\title{
Heterogeneous Network Performance Improvement Using Proposed OLRED and OLWRED Strategies
}

\author{
Wisam Mahmood Lafta, Saba Qasim Jabbar, Dheyaa Jasim Kadhim, and Guangzhi Ma
}

\begin{abstract}
The congestion and queue delay are the critical problems that may face the heterogeneous networks, because of the huge data broadcasting in every network segment. So the selection of suitable strategy to solve these two major problems is considered in this work. Therefore, this paper proposes two new strategies based on optimized link state routing protocol (OLSR), random early detection (RED) and weight random early detection (WRED), which we defined them as OLRED and OLWRED respectively. These two proposed strategies are applied and examined the performance of the heterogeneous networks in terms of three important metrics: throughput, utilization and queue delay. Our simulation results are done using OPNET and they show that the proposed strategies are improved the QoS for the heterogeneous networks and reducing the congestion with high network productivity.
\end{abstract}

Index Terms-Heterogeneous networks, OLSR, RED, WRED, queue delay, congestion.

\section{INTRODUCTION}

The demand of transmitting big data (video, FTP, audio) with high speed depends on applications in all kinds of networks (wired and wireless), the wire networks need to apply many algorithms by used different strategies to meet of requiring quality of service (QoS). The "Best-effort" quality of service is achieved based on FIFO (First In/First Out) queue management [1]. The control on congestion and keeping on high-speed communication is utilized large amount of bandwidth with less queue delay and high throughput by multiple sources is a big challenge for network type [2]. TCP is one of the most widely used on networks as transmission protocol and on the Internet. The basic idea of congestion avoidance strategies is that, when a router's buffer utilization reaches a certain threshold, it will drop packets, according to a certain probability, to notify TCP sources to reduce the congestion window, so as to achieve the aim of congestion avoidance [3], [4].

In order to solve the problem of congestion control, there are many suggestions in different types of approaches as

Manuscript received July 12, 2016; revised October 12, 2016.

Wisam Mahmood Laft and Guangzhi Ma are with the School of Computer Science and Technology, Huazhong University of Science and Technology, Wuhan 430074, P.R. China (e-mail: wisamone@yahoo.com, maguangzhi@hust.edu.cn).

Saba Qasim Jabbar is with School of Electronic Information and Communication Engineering, Huazhong University of Science and Technology, Wuhan 430074, P.R. China (e-mail: shura2007515@yahoo.com).

Dheyaa Jasim Kadhim is with the Electrical Engineering Department, University of Baghdad 47024, Baghdad, Iraq (e-mail: deya_naw@yahoo.com). shown in [1], [2], [4]-[7]: discordant connection, link layer, end-to-end, and network cooperation. The basic idea of discordant-connection approach [7] is to isolated connection at the base station between wired and wireless network. The base station serves as a relay node to isolated congestion control functionality on wireless links from that on wired networks. This strategy needs a big buffer to store and forward packets to the mobile host. In another side the approaches [5], [6], [8] used automatic repeat request (ARQ) this technical used in IEEE 802.11 MAC and in 802.16 MAC (WiMAX), to reduce wireless losses and to recover transmission error locally by retransmitting the lost frame at the link layer, and also LTE protocols. To implement the two approaches mentioned above the control load is huge as the base station needs to keep a significant amount of state information for each TCP connection.

Instead of the support from the base station, the network cooperation approach [5]-[7] requires the assistance from intermediate routers to send the information about network condition to end-systems for the improvement of congestion control efficiency in the presence of wireless losses. The intermediate routers do not require per-flow states.

The packet loss is used as indicator of network congestion, that it will be happened when the congestion control mechanism is implemented in a traditional TCP. That means it is not suitable to traveling packets on nodes over heterogeneous networks,it will lead to decrease the performance because of the incorrect reduction of the congestion window [5], [8]. The distortion of the signal is most mistake have been significantly underserved caused the error bit transfer and packet loss in the wireless networks, therefore, the congestion and queue delay with less throughput will appear however, the timeouts is the main problem a traditional TCP over wired networks [7], [9]. This is a big challenge between wire and wireless in heterogeneous networks lead to degraded performance because of the looseness reduction of the congestion window [9].

The paper is organized as follows: summarization of the two strategies appears in Section II and presents the proposed work. The simulation environments and results are presented in Section III. Finally, the conclusion of this work is given in Section IV.

\section{SYSTEM MODEL}

The TCP protocol is useful in wired networks to solve the problem of congestion, as packet timeouts are most of the time caused by congestion. Including, the TCP in wireless links is subjected to a lot of error bit and packet loss because the type of connection, this is totally inappropriate in wireless 
networks. In practice a lot of error bits and packet loss due to fading, distortion, handoff and other wireless broadcast effects [6], [10]. Currently, almost TCP congestions are caused by timeouts not by transmission errors, this happened in wired networks. However, this behavior does not stand for wireless networks as they suffer from packet loss rates and high bit error. TCP congestion protocol is designed suitable for wired networks because it is more reliable than the wireless network, it is impossible for the source discrimination between congestion loss or error loss [6], [10]. So we should implement the algorithm with a wireless network to avoid or reduce congestion and error loss. This work will apply OLSR routing algorithm and RED/WRED strategies with TCP protocol to let make it more suitable for work with wire and wireless networks (i.e. heterogeneous network).

\section{A. RED/WRED Strategies}

The basic idea of congestion avoiding strategies or mechanisms, when buffer utilization of a router's reaches a certain threshold, it will drop packets, achieve the aim of congestion avoidance [10]. RED and WRED are used to congestion avoidance strategies, these two strategies are discussed and analyzed in [1], [2], [3], [5]. The calculation of RED operators are depending on the average of queue size $\left(\mathrm{Q}_{\mathrm{avg}}\right)$ and a drop probability $\left(\mathrm{P}_{\mathrm{d}}\right)$ based on the instantaneous queue size and a weight factor $\mathrm{w}$. In addition, RED maintains two thresholds of queue size $\left(\mathrm{Q}_{\min }\right)$ and $\left(\mathrm{Q}_{\max }\right)$. The random early detection (RED) algorithm is becoming the main factor standard for congestion avoidance in the packet switched networks or on the Internet as mentioned in RFC2309 [9].

$\mathrm{W}_{\mathrm{q}}$ is the weight the value range is from 0 to $1, \mathrm{q}$ is the actual queue length of sampling measurement. The drop probability is calculated according to average queue length. WRED strategy uses the random early detection factors additional to the weight to make it more efficient and robust from the strategy of RED in process, WRED is making of RED and Priority Queuing, it sets different minimum threshold $\mathrm{MIN}^{\text {th }}$, maximum threshold $\mathrm{MAX}^{\text {th }}$ and the highest drop probability $\mathrm{P}_{\max }$ for packets with different types of services. WRED will drop packets selectively depending on the priority. Then, strong recommendations for testing, standardization and widespread deployment of AQM in routers are made to improve the performance of today's Internet.

\section{B. Proposed OLRED and OLWRED Strategies}

The main challenge in wireless network with the node's mobility is the congestion. The congestion occurs when the source sending data more than the buffer capacity almost about to be overflow. In wireless ad hoc networks, the routing protocols are categorized into three types i) proactive, ii) reactive and iii) hybrid, which can also classified the routing protocols into two another categories i) congestion-control routing and ii) congestion non-control. The control routing protocol occurs through the network when the packets transfer from source to destination; this problem will increase delay and reduce throughput. Proactive Routing is based upon a table driven approach. Each node has to maintain routing information to the other nodes in the network. This information is usually stored in a number of different tables which are updated periodically and/or upon the detection of changes within the network. Which information is kept and how it is exchanged varies from the used routing protocols. The two acknowledged routing protocols from the MANET working group officially which they are out of this category are Topology Based Reverse Path Forwarding and the OLSR [11]-[16].

Fig. 1 below shows three different retransmission approaches; first one is shown in Fig. 1a which there is no using of OLSR routing protocol, while Fig. 1b shows the same network with using OLSR routing protocol which reduces the number of retransmissions and Fig. 1c shows how our proposed strategies will improve the retransmission process and how these strategies are reduced the congestion.

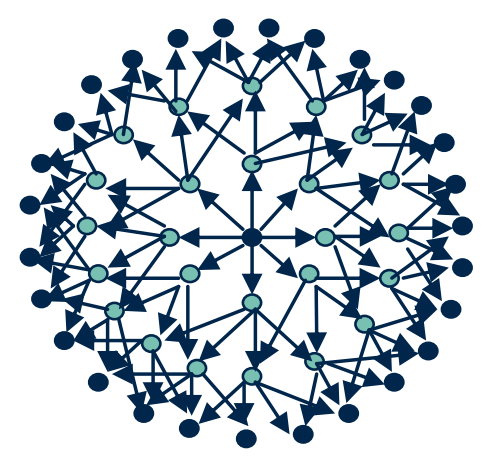

24 retransmissions to diffuse a message up to 3 hops

Retransmission node

a)

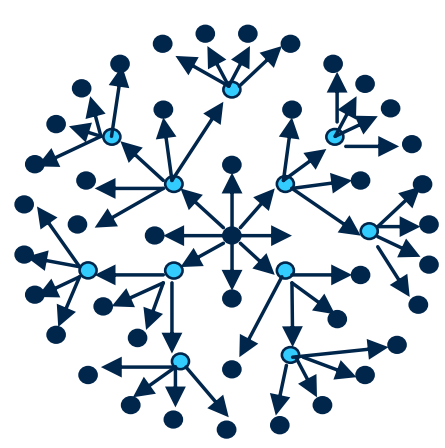

11 retransmission to diffuse a message up to 3 hops

Retransmission node - MPR

b)

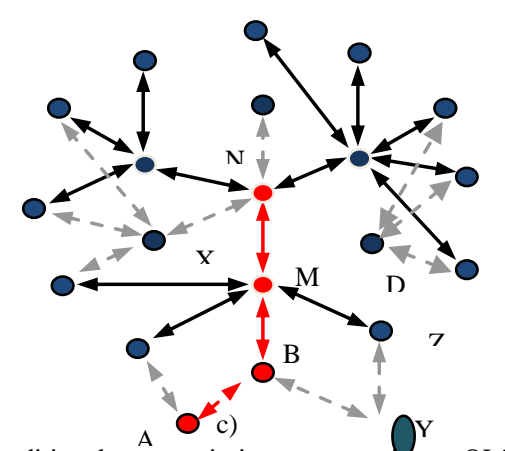

Fig. 1 a). Traditional retransmission process without OLSR; b). Retransmission process with OLSR; c). Retransmission process with proposed strategies. 
The Algorithm's steps of our two proposed strategies is OLRED and OLWRED are described in details as shown below:

\section{Algorithm of OLRED}

\section{Begin}

\{

The first step selects the best path Broadcast (demand data) from source to destination (check for update interval).

Each node expands a spanning tree

If ( node 1 cost > nod2 cost )

Select nod 2 cost

Else

Select nod 1 cost

Examine all links available from the source to the target (over once in a node).

Select the best link (update interval)

Insert link in table - driven

The second step applies Multi-Point Relaying (MPR).

Send signal to Each node floods status of its links (periodically)

Wait to Each node re-broadcasts link state information ( received from its neighbors)

Each node keeps track of link state information received from other nodes (depend on select paths in driven table).

Only selected neighbors (MultiPoint Relays, MPRs) retransmit messages

Select MPRs such that they cover all 2-hop neighbors

2-hop neighbors taken( send acknowledgment) from neighbors' HELLO messages

Update link in table - driven

Third step with queue management

Arrival packet to gateway (router)

Take the MIN threshold and MAX threshold periodically (Apply RED strategy).

Calculate average queue size avg

$=\left(1-\mathrm{W}_{\mathrm{q}}\right) \times \operatorname{avg}+\mathrm{W}_{\mathrm{q}} \times \mathrm{q}$

- Compare the average queue size(Ave) with Min and Max threshold periodically according to

If avg > min threshold and Min<avg > max then

Enter in queue (FIFO)

Else

If Aver >Max then

The queue will be dropped the random packet

Else

Calculate the Packet dropping probability

Return to beginning

\}

End

\section{Algorithm of OLWRED}

Begin

\{

The first step selects thebest path Broadcast (demand data) from source to destination (Pass-through reference to all nodes).

Each node expands a spanning tree

If (node 1 cost $>\operatorname{nod} 2$ cost )

Select nod 2 cost

Else

Select nod1cost

Examine all links available from the source to the target (over once in a node).

Select the best link (shortest and lowest cost) Insert link in table - driven

The second step applies Multi-Point Relaying (MPR)

Send signal to Each node floods status of its links (periodically)

Wait to Each node re-broadcasts link state information ( received from its neighbors)

Each node keeps track of link state information received from other nodes (depend on select paths in driven table).

Only selected neighbors (MultiPoint Relays, MPRs) retransmit messages
Select MPRs such that they cover all 2hop neighbors

2-hop neighbors taken( send acknowledgment) from neighbors HELLO messages

Update link in table - driven

Third step with queue management

Arrival packet to gateway (router)

Take the Min threshold and Max threshold periodically (Apply

WRED strategy).

Calculate average queue size avg

$=\left(1-\mathrm{W}_{\mathrm{q}}\right) \times \mathrm{avg}+\mathrm{W}_{\mathrm{q}} \times \mathrm{q}$

and the highest drop probability $\mathrm{P}_{\max }$

Compare the average queue size(Ave) with Min and Max

threshold periodically according to

If $\mathrm{avg}>\mathrm{min}$ threshold and Min<avg > max then

Enter in queue (FIFO)

Else

Ifavg>Max then

The queue will be dropped the random packet periodically

Else

Calculate the Packet dropping probability

Return to beginning

\}

End

The flowcharts of these two algorithms are shown below in Fig. 2 and Fig. 3 respectively. These flowcharts contains two different environments (wired and wireless) which we consider as a heterogeneous network starting from wireless environment to select the optimal link for the sender to the target receiver.

The main difference between OLRED and OLWRED can be summarized as the OLRED new strategy selects optimal routing link periodically and calculates the average of queue size one time, while the new strategy OLWRED selects optimal routing link and calculates the average of queue size periodically. This difference makes OLWRED strategy comprises more sensitive to the storage area (average queue size) and also makes it more robust towards the number of arriving packets in queue to reduce from dropping queue packet.

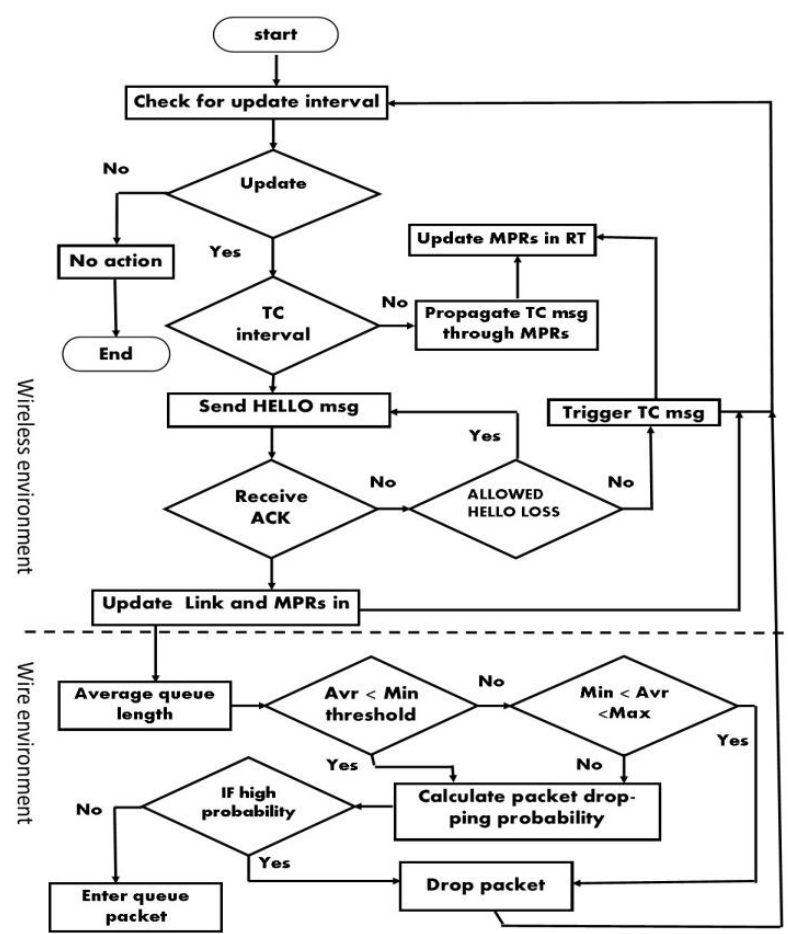

Fig. 2. OLRED strategy flowchart. 


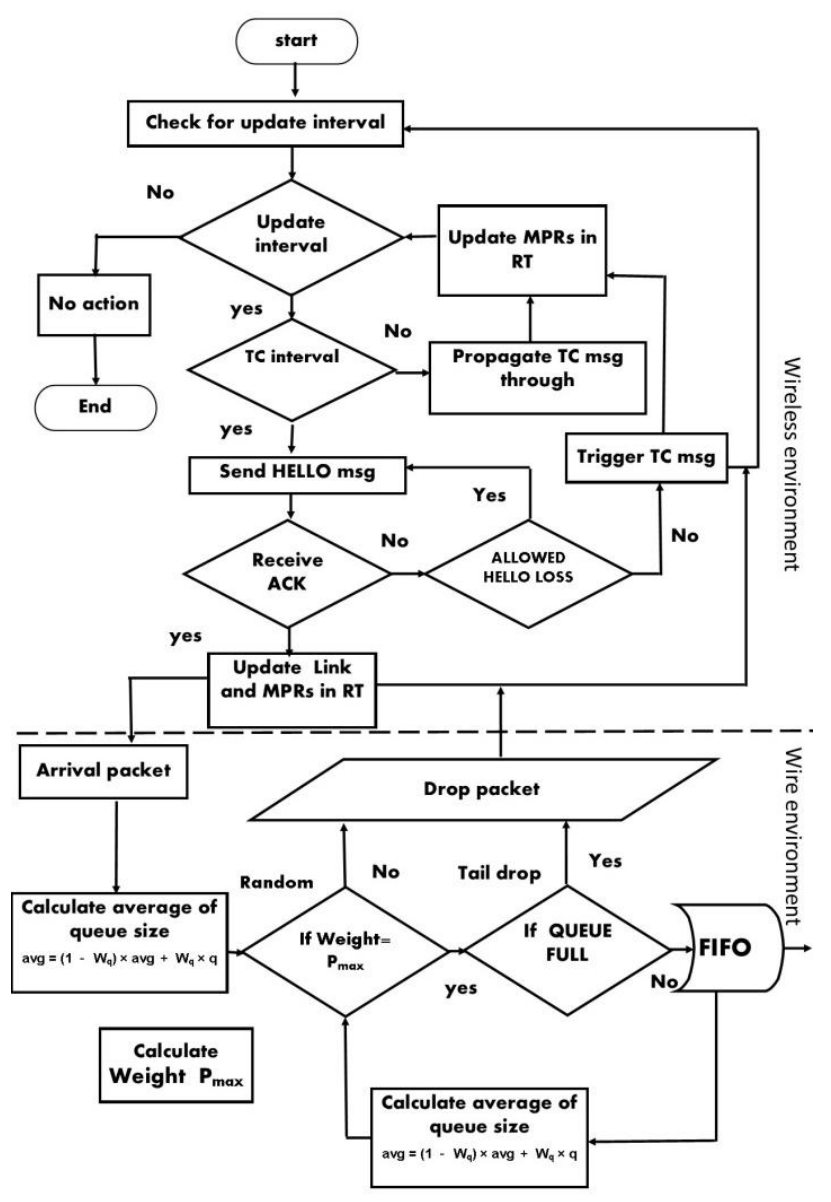

Fig. 3. OLWRED strategy flowchart.

\section{OPNET SimULATION RESULTS}

We implement our simulation in $2500 \mathrm{~m} * 5000 \mathrm{~m}$ area with 24 mobile nodes connected randomly by wireless distributed links to access point, the access point is connected by wire type (base100) with router which is connected also to second router by wire (PPP_DS1) link (1.53Mbps), while the second router is connected to a switch (ethernet16_switch) and the switch is connected to the server by the base 100 wire. The devices should be set up as: duration $=600$ seconds, to transfer data $(600 \mathrm{Gbit} / \mathrm{second})$ and $(5 \mathrm{Kpacket} / \mathrm{second})$. Table I shows the main contents of our system model in the simulation environment.

TABLE I: SIMULATION ENVIRONMENT

\begin{tabular}{|c|c|c|c|c|c|}
\hline Element & No. & Name & connect & $\begin{array}{c}\text { Type } \\
\text { of app. }\end{array}$ & $\begin{array}{l}\begin{array}{l}\text { Type of } \\
\text { service }\end{array} \\
\end{array}$ \\
\hline Router & 2 & $\begin{array}{l}\text { Ethernet } \\
\text { slep4 }\end{array}$ & wire & FTP & Best-efforts \\
\hline Switch & 1 & & wire & FTP & Best-efforts \\
\hline $\begin{array}{c}\text { Access } \\
\text { point }\end{array}$ & 1 & & $\begin{array}{l}\text { Wire/wi } \\
\text { reless }\end{array}$ & FTP & Best-efforts \\
\hline Server & 1 & & wire & FTP & Best-efforts \\
\hline $\begin{array}{c}\text { Mobile } \\
\text { node }\end{array}$ & 24 & $\begin{array}{l}\text { Wlan_w } \\
\text { kst_adv }\end{array}$ & wireless & FTP & Best-efforts \\
\hline App. & 1 & $\begin{array}{l}\text { App. } \\
\text { def. }\end{array}$ & ----------- & FTP & Best-efforts \\
\hline profile & 1 & $\begin{array}{c}\text { App. } \\
\text { profile }\end{array}$ & ---------- & FTP & Best-efforts \\
\hline QOS & 1 & IP QOS & ----------- & FTP & Best-efforts \\
\hline
\end{tabular}

To implement our proposed strategies, we need to consider the following five scenarios for our simulation as follows:

- Scenario 1 (Normal demand): The maximum queue size (85), the weight for the queue (9), the MIN threshold (100), MAX threshold (200) and packet size (500000 bps) with interval time (0.05).

- Scenario 2 (RED strategy): The maximum queue size (85), the weight for the queue (9), the MIN threshold (5), MAX threshold (15) and packet size (500000 bps) with interval time (0.05).

- Scenario 3 (WRED strategy): The maximum queue size (85), the weight for the queue (12), the MIN threshold (5), MAX threshold (15) and packet size (500000 bps) with interval time (0.05).

- Scenario 4 (the new OLRED strategy): The maximum queue size (85), the weight for the queue (9), the MIN threshold (5), MAX threshold (15) and packet size (500000 bps) with interval time (0.05).

- Scenario 5 (the new OLWRED strategy): The maximum queue size (85), the weight for the queue (12), the MIN threshold (5), MAX threshold (15) and packet size (500000 bps) with interval time (0.05).

The set of weight values in queue size is changed from 9 to 12 to let queue more sensitive and the result highly robust, also to be suitable with a number of packets, because the number of packet changes every time. The summary of these five scenarios are shown below in Table II.

To measure the results in the OPNET simulation, we need to define the following three parameters:

1) Queue delay: It is the job time which waits in a queue until it can execute, this term is almost used in reference to routers. Also it can be defines as the time spending in the router to be processed and transmitted.

2) Throughput: This term is used to measure the performance of a network, it is the number of packets successfully delivered per unit time and controlled on available bandwidth.

3) Utilization: This term is used to host a larger amount of traffic on the different networks, thus reducing operation cost and being the foundation for hosting the exponential growth of modern networks.

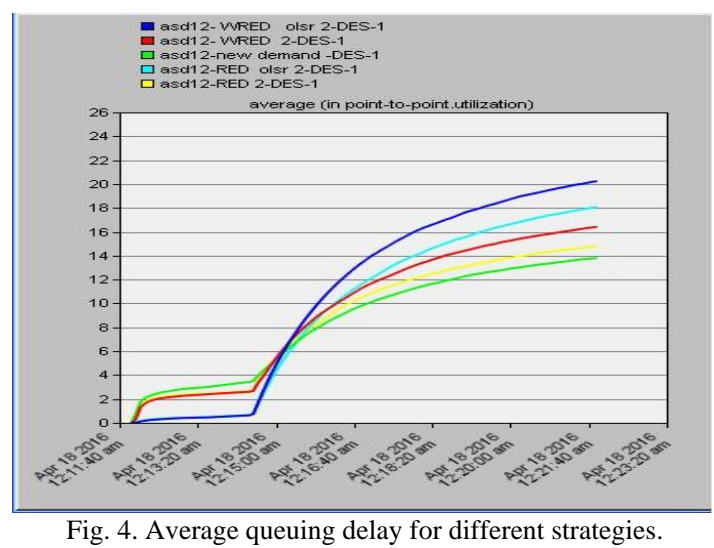

Fig. 4 shows that the queue delays acquired by the four strategies are different when (New Demand, RED, WRED, OLRED and OLWRED), if queue delay is increased, then it will effect on the speed of network and it considers as the main factor for the emergence of congestion. In this case we should be minimized from queue delay parameter. To ensure there is no bottleneck in the network, because it is the first risk index occurrence of congestion. In our proposed new 
strategy OLWRED, we can note the less curve in Fig. 4, we

less one is better and it is achieved at OLWRED strategy. compare the average of queue delay with other curves. The

TABLE II: SUMMARY OF THE SiMULATION'S FIVE SCENARIOS

\begin{tabular}{|c|c|c|c|c|c|c|c|}
\hline & queue pro. & Max queue & Weight & $\begin{array}{c}\text { Min } \\
\text { Th. }\end{array}$ & $\begin{array}{c}\text { Max } \\
\text { Th. }\end{array}$ & EXP & Packet size/bps \\
\hline Sc. 1 & FIFO & 85 & 9 & 100 & 200 & 0.05 & 500000 \\
\hline Sc. 2 & FIFO & 85 & 9 & 5 & 15 & 0.05 & 500000 \\
\hline Sc. 3 & FIFO & 85 & 12 & 5 & 15 & 0.05 & 500000 \\
\hline Sc. 4 & FIFO & 85 & 9 & 5 & 15 & 0.05 & 500000 \\
\hline Sc. 5 & FIFO & 85 & 12 & 5 & 15 & 0.05 & 500000 \\
\hline
\end{tabular}

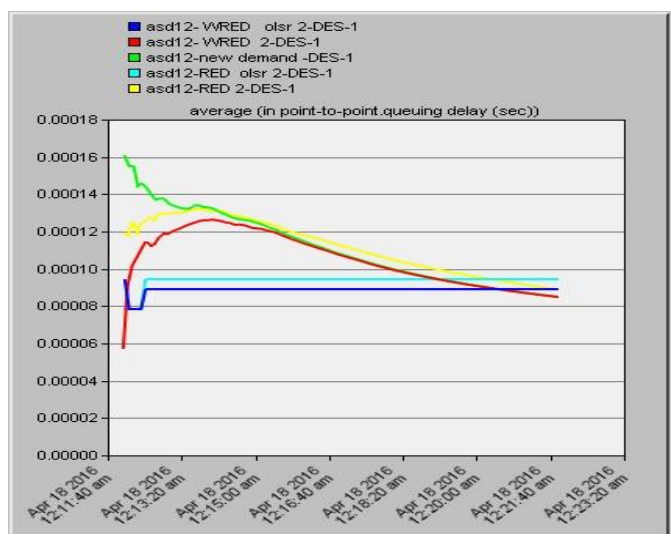

Fig. 5. Average throughput for different strategies.

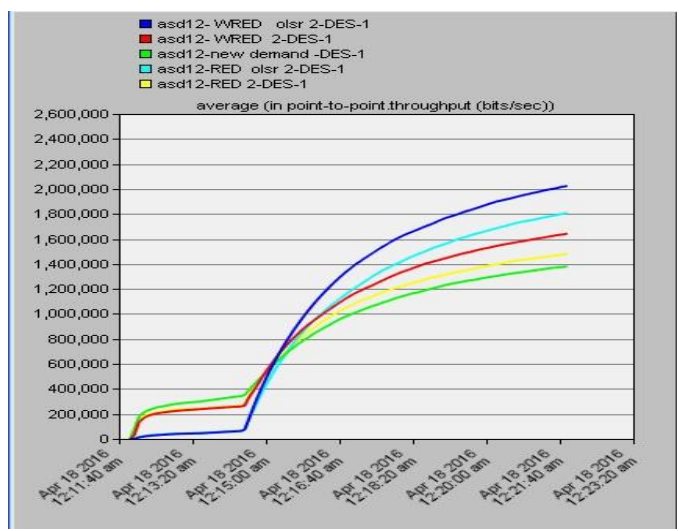

Fig. 6. Average utilization for different strategies.

Beside the queue delay factor, there are other factors to measure the efficiency of the network, to denote the network efficiently by knowing packet loss ratio, which is inversely proportional to the productivity of the network, and represented by throughput, in other words, it must be efficient, high productivity of the network with a little delay in queue delay, that achieved in OLWRED strategy. The new strategy OLWRED shows the network performance with high throughput appearing clearly in Fig. 5 below.

The another important performance index is utilization, from this parameter, we will get to know how characterize network resources properly, and it is also an important factor to indicate the effectiveness of the network performance, and by maintaining the ratio of high-performance network makes a high coefficient of performance, this is achieved in a curve of utilization in new strategy OLWRED higher from other as shown in Fig. 6 below.

\section{CONCLUSIONS}

The result of our simulation in different scenarios reduced congestion happened by decreasing of queue delay and increasing the performance and utilization in heterogeneous networks, that mean we avoidance congestion and let the network more speed to transmit big data, in this heterogeneous networks we apply two new strategies OLRED/ OLWRED strategies with TCP protocol. In the above results proved that the search feature OLWRED got the best results and performance Compared with OLRED and others.

\section{REFERENCES}

[1] X.-J. Xue et al., "Simulation study of RED/WRED mechanism based on OPNET," presented at International Conference on Mechatronics, Electronic, Industrial and Control Engineering (MEIC 2015).

[2] M. Shweta and N. Jain. "Effective impact of ECN with RED to control heavy congestion," International Journal of Computer Science and Information Technologies, vol. 3, no. 3, pp. 4290-4293, 2012.

[3] D. Vivek, P. Sarode, and S. Sarode. "EDCAM-early detection congestion avoidance mechanism," International Journal of Computer Application, vol. 7, no. 2, pp. 11-14, September 2010.

[4] Youssef, "TCP congestion control scheme for wireless networks based on TCP reserved field and SNR ratio," arXiv preprint arXiv:1207.1098, 2012.

[5] L. W. Mahmood et al., "Performance evaluation of heterogeneous network based on RED and WRED," Indonesian Journal of Electrical Engineering and Computer Science, vol. 3, no. 3, 2016, pp. 540-545.

[6] S. Mascolo, C. Casetti, M. Gerla, M. Y. Sanadidi, and R. Wang, "TCP westwood: Bandwidth estimation for enhanced transport over wireless links," in Proc. the 7th annual international conference on Mobile computing and networking, pp. 287-297, ACM, 2001.

[7] G. Dinesh and D. S. Tomar, "Dynamic queue and TCP based multipath congestion control scheme for wired network," International Journal of Computer Application, vol. 123, no. 10, pp. 45-50, August 2010.

[8] S. A. Mondal and F. B. Luqman. "Improving TCP performance over wired-wireless networks," Computer Networks, vol. 51, no. 13, pp. 3799-3811, 2007.

[9] S. Chen, X. Hei, J. Zhu, and B. Bensaou, "Congestion control in the wired-cum-wireless internet," Heterogeneous Wireless Access Networks: Architectures and Protocols, 2009, p. 333

[10] A. Bakre and B. R. Badrinath, "I-TCP: Indirect TCP for mobile hosts," in Proc. the 15th International Conference on Distributed Computing Systems, IEEE, 1995.

[11] J. S. Fan, S. S. Wang, and H. F. Hsiao, "Wireless-aware congestion control for transmission over heterogeneous networks," in Proc. the International Conference on Wireless Networks (ICWN), 2014.

[12] M. Shweta and N. Jain, "Effective impact of ECN with RED to control heavy congestion," International Journal of Computer Science and Information Technologies, vol. 3, no. 3, 2012, pp. 4290-4293.

[13] R. Ramanathan and J. Redi, "A brief overview of ad hoc networks: challenges and directions," IEEE communications Magazine, vol. 40, no. 5, pp. 20-22, 2002.

[14] D. Mitzel, "Overview of 2000 IAB wireless internetworking workshop,” No. RFC 3002, 2000.

[15] Y. S. Yen, H. C. Chang, R. S. Chang, and H. C. Chao, " Routing with adaptive path and limited flooding for mobile ad hoc networks," Computers and Electrical Engineering, vol. 36, no. 2, pp. 280-290, 2010.

[16] H. Balakrishnan, V. N. Padmanabhan, S. Seshan, and R. H. Katz, "A comparison of mechanisms for improving TCP performance over wireless links," IEEE/ACM Transactions on Networking, vol. 5, no. 6 , pp. 756-769, 1997. 


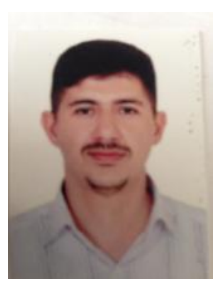

Wisam M. Lafta was born in Baghdad, Iraq. He got the BSc in computer science at University of Technology. He is currently an MS student at Huazhong University of Science and Technology in China. He is worked at Information Technology and Communication Center, University of Technology, Baghdad, Iraq. He has some important published papers in international journals.

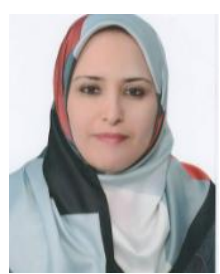

Saba Q. Jabbar received the BSc in computer engineering at Baghdad University; the MSc in communication and information engineering at Huazhong University of Science and Technology in China. She is currently the PhD student at Huazhong University of Science and Technology in China. She works as a faculty member in the Computer Engineering Department, Baghdad University. She has some important published papers in international journals and conferences.

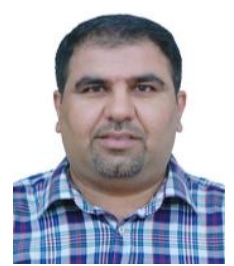

Dheyaa J. Kadhim received the BSc in electrical engineering at Baghdad University; the MSc in electrical engineering at Baghdad University; the $\mathrm{PhD}$ in communication and information engineering at Huazhong University of Science and Technology in China. He is currently faculty member in the Electrical Engineering Department, Baghdad University. He is one member of Iraqi Engineers Society, editor and reviewer at some international journals.

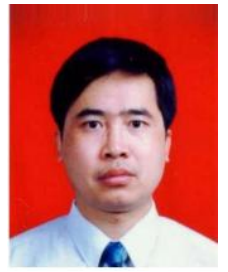

Guang-Zhi Ma is an associate professor in computer science and technolog at Huazhong University of Science and Technology, China. He received his M.S and $\mathrm{Ph} . \mathrm{D}$. in computer science from Huazhong University of Science and Technology. He had been trained in North Illinois University for IBM S390 System. His research interests include decision support using data warehousing and data mining, computer aided diagnose using neural network, multiple object optimization using genetic algorithm, and database and network applications in health information systems. 